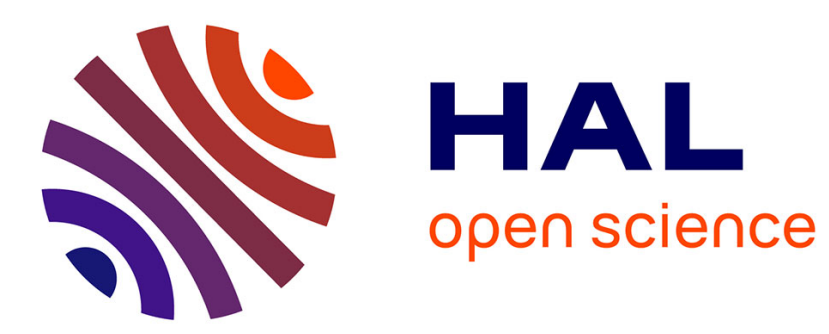

\title{
Religions transnationales et migrations : regards croisés sur un champ en mouvement
}

\author{
Sophie Bava, Stefania Capone
}

\section{To cite this version:}

Sophie Bava, Stefania Capone. Religions transnationales et migrations : regards croisés sur un champ en mouvement. Autrepart - Revue de sciences sociales au Sud, 2010. ird-02067964

\section{HAL Id: ird-02067964 \\ https://hal.ird.fr/ird-02067964}

Submitted on 14 Mar 2019

HAL is a multi-disciplinary open access archive for the deposit and dissemination of scientific research documents, whether they are published or not. The documents may come from teaching and research institutions in France or abroad, or from public or private research centers.
L'archive ouverte pluridisciplinaire HAL, est destinée au dépôt et à la diffusion de documents scientifiques de niveau recherche, publiés ou non, émanant des établissements d'enseignement et de recherche français ou étrangers, des laboratoires publics ou privés. 


\title{
Religions transnationales et migrations : regards croisés sur un champ en mouvement
}

\author{
Sophie Bava*, Stefania Capone**
}

Depuis quelques années, les religions des migrants ont acquis une nouvelle visibilité et la fréquentation croissante de leurs lieux de culte témoigne de la place qu'elles occupent au cœur des agglomérations urbaines. Les modèles religieux et identitaires se multiplient et se répandent, si bien qu'il semble parfois difficile de saisir leur évolution, l'étendue des pratiques rituelles qui leurs sont associées, ainsi que les motivations des fidèles. Pourtant, derrière le renouvellement religieux de ce début du $\mathrm{XXI}^{\mathrm{e}}$ siècle, nous observons des logiques et des processus religieux historiquement ancrés, dont la diffusion peut se comprendre par la circulation des individus et plus concrètement par les migrations contemporaines. Par leur nature même, les migrations ravivent et reconfigurent l'offre religieuse des grandes villes de transit ou d'installation. La circulation des migrants suscite de nouvelles dynamiques religieuses et en réactive d'anciennes. Ainsi, quand ce ne sont pas les Églises et les ONGc qui viennent à la rencontre des nouveaux arrivants, ce sont ces derniers qui créent leurs propres espaces de prière, en s'appuyant sur des réseaux religieux déjà existants ou en construisant de nouveaux espaces religieux.

Si les religions des migrants renvoient automatiquement à des religions « ethniques », communautaristes, étrangères, qui doivent trouver leur place au sein de la société d'accueil, les religions transnationales ne sont pas forcément liées aux flux migratoires et les ressources identitaires qu'elles véhiculent sont souvent mobilisées par des membres de la nouvelle société, qui se «convertissent » à des religions qui ne faisaient pas nécessairement partie du paysage religieux local. Dans le transnational, la mobilité du religieux n'est pas seulement «passage des frontières » et "parcours migratoires », le mouvement n'étant pas toujours un préréquisit de toute action transnationale. « Transmigrants » [Glick-Schiller, Basch et Szanton Blanc, 1994 ; Guarnizo, 1997] ou « sédentaires », c'est-à-dire des individus qui, après avoir migré, sont profondément ancrés dans la société d'accueil,

\footnotetext{
* Socio-anthropologue, chargée de recherche à l'IRD/LPED, Université du Caire, sophie.bava@ird.fr

** Anthropologue, directrice de recherche au CNRS, Laboratoire d'ethnologie et de sociologie comparative (LESC - UMR 7186), Université de Paris Ouest Nanterre La Défense. stefania.capone@ mae.U-paris10.fr
} 
ils sont tous intégrés dans des réseaux qui, comme nous le verrons, les relient par les flux de personnes, de marchandises ou d'informations - à leur pays d'origine.

Alors que, jusqu'aux années 1990, le lien entre migration et religion n'était pas encore au cœur des études sur la migration, l'intensification et la redéfinition des flux migratoires, combinées à une visibilité plus grande de la «religion des migrants », ont poussé les chercheurs à s'intéresser au rôle des migrations dans la diffusion et la création religieuse, ainsi qu'à la place des religions dans la compréhension des processus migratoires. C'est aux États-Unis qu'une nouvelle approche commence à se développer à partir des années 1990, conjuguant migrations, religions et transnationalisme. Des nouvelles pistes de recherche sont ainsi ouvertes par une génération de chercheurs anglophones, dont les apports aux études sur les « religions en migration » sont analysés dans ce numéro par Stefania Capone.

\section{La relation migration-religion vue de la France ${ }^{1}$}

En France, c'est l'identité religieuse de l'immigré dans le pays d'accueil qui a retenu l'attention des chercheurs dès le début des années 1980, au détriment, souvent, de la construction de son parcours religieux. La combinaison de ces approches du fait religieux allait permettre de mieux comprendre comment l'Autre se construit et « s'intègre », en s'adaptant à sa nouvelle société. Ainsi, après les grandes enquêtes commanditées par l'État dans les années 1980, afin de connaître les conditions de vie des travailleurs immigrés [ $c f$. Kuczynski et Razy, 2009], les recherches autour de l'avènement d'une société multiculturelle, initiées par les sociologues et les politologues, vont intégrer la question de la religion de l'Autre «immigré ».

Pendant longtemps, le facteur religieux n'avait guère été pris en compte dans la compréhension des processus migratoires. Les premières études dans les foyers de travailleurs migrants, portant sur l'islam en banlieue et les salles de prière, ouvrent ce champ de recherche en France en mettant l'accent sur la nécessité pour les immigrés de pratiquer leur culte décemment et de vivre leur culture pleinement au sein de la société française. C'est également à cette même période que des sociologues et anthropologues, comme Jacques Barou [1978], A. Moustafa Diop [1989] et Catherine Quiminal [1991], commencent à mener des recherches, non plus seulement sur les immigrés en France, mais aussi sur les deux espaces de la migration : les sociétés de départ et celles d'accueil. Cependant, l'immigré n'était pas encore considéré comme un acteur à part entière de sa religion. De même, la diversité de l'offre religieuse engendrée par les migrations était rarement prise en compte par ces recherches. L'immigration était souvent appréhendée comme définitive et la religion comme l'une des multiples valeurs transplantées devant s'adapter au modèle laïque français.

1. Pour un approfondissement de cette question, voir Bava [2011]. 
Dans la France de la fin des années 1980, le débat va ainsi se focaliser sur le thème «minorités ethniques et identités religieuses ». C'est surtout l'islam qui mobilise les analyses, et tout particulièrement la question de l'islam face à la laïcité ${ }^{2}$. Cette question, partagée par les acteurs du monde politique et les travailleurs sociaux, relève plus d'une peur face à des minorités religieuses «incontrôlables » que d'une analyse des différentes formes de laïcité possibles [Baubérot, 1994]. Si les recherches sur les migrations et les religions en France ont du mal, encore aujourd'hui, à se départir du débat sur l'intégration, de nombreuses études, initiées en grande partie par des anthropologues à la fin des années 1990, ont ouvert le champ à une connaissance de la religion telle qu'elle est vécue par ses acteurs. Ces recherches sollicitent une approche des religions en termes de pratiques sociales, pratiques cultuelles et organisation de lieux de cultes [Geisser, 2001 ; Bava, 2002 ; Kuczynski, 2002 ; Fancello, 2005] ; elles interrogent les conditions de production de viande halâl, l'organisation de l'Aïd el Kebîr dans l'espace urbain français [Bonte, Brisebarre et Gokalp, 1998 et 1999] et le sens que les croyants donnent à leurs pratiques religieuses [Saint-Blancat, 1997] ; elles analysent les réseaux économiques de la religion [Bava, 2003], les parcours et carrières religieuses, la construction des identités au sein des pentecôtismes en migration [Fancello, 2006], sans oublier l'expansion des Églises africaines dans les grandes métropoles françaises à partir des années 2000 [Mary, 2008].

Ce qui motive ces recherches n'est plus l'approche en termes d'intégration mais bien l'analyse des religions et des cultes réimplantés ou recréés par les migrants, grâce à une connaissance fine des (dis)continuités, historiques et cultuelles, entre pays d'origine et pays de migration. Travailler la question de la religion dans la migration, particulièrement une migration observée à partir de ses fluidités tout autant que de ses permanences, nécessite d'accepter cette dynamique d'échange et d'aller-retour entre la soi-disant «authenticité » accordée aux religions dans leurs sociétés d'origine et les itinéraires religieux de chaque migrant et de chaque mouvement étudié.

Grâce au croisement des études des anthropologues, des sociologues anglosaxons et des sociologues de l'immigration en France, une troisième voie s'est ouverte autour d'une socio-anthropologie qui interroge les liens tissés par les migrants entre espaces traversés et espaces d'installation. Les chercheurs dépassent les questions d'assimilation, d'intégration et/ou de ruptures et diversifient les méthodes d'observation, en mettant en évidence les dynamiques qui permettent aux migrants d'exister socialement en tant que groupe, grâce à la création de leurs propres réseaux économiques, sociaux, imaginaires entre plusieurs territoires. En donnant un nouvel élan à l'analyse croisée des migrations et des religions, les chercheurs vont alors s'intéresser aux constructions religieuses mises en place par les migrants, ainsi qu'à leurs appartenances religieuses. Cette vague de recherches

2. Sur l'islam des migrants en France et en Europe, voir, entre autres, Diop et Kastoryano [1991], Andezian [1983 et 2001], Dassetto et Bastenier [1991], Saint-Blancat [1995 et 1997], Cesari [1994]. 
a été largement influencée par une sociologie du transnational, de la mobilité et de la circulation des acteurs.

En effet, à partir du début des années 1990, les études sur les migrations transnationales intègrent progressivement une approche transculturelle dans la compréhension des parcours migratoires et, en parallèle, l'approche transnationale des phénomènes religieux apporte une nouvelle compréhension des religions « en migration ». Un renouvellement théorique s'est donc opéré autour de la notion de migration. Aujourd'hui, la migration est moins envisagée comme une rupture, comme une succession de lieux ; elle n'est plus interprétée comme un « déracinement » ou une « acculturation ». Les études anglophones et francophones portant sur les entrepreneurs migrants ont introduit la notion de transnationalisme [Basch, Glick-Schiller et Szanton Blanc, 1994] comme un nouvel outil pour appréhender le champ migratoire. On parlera alors de migrants transnationaux, de «transmigrants », de migrants pris dans une mobilité pendulaire ou circulaire [Tarrius, 1989]. Il s'agit, dans ce contexte, de décrire le territoire relationnel que l'étranger met en scène et les territoires qu'il connecte dans un espace transnational, connexions qui peuvent être à caractère économique, mais aussi culturel ou religieux. En ce sens, la migration n'est plus abordée seulement en termes de coûts, mais plutôt en termes de ressources, de compétences et de bénéfices produits par ces allers-retours entre deux ou plusieurs pays. Nous verrons que ce « savoir circuler » [Tarrius, 1996] et cette mobilité peuvent être analysés comme un phénomène social créateur de richesses mais également producteur d'imaginaires.

\section{Variations religieuses et pluralité des parcours migratoires}

Les articles présentés dans ce numéro apportent, chacun à sa manière, de nouveaux éclairages sur ce débat. Si les disciplines varient, les approches de type ethnographique, choisies par la plupart des auteurs, relèvent d'une méthode d'enquête multi-localisée, la plus à même de rendre compte de la fluidité des pratiques religieuses et de la circulation des migrants. Au-delà des disciplines et des aires géographiques privilégiées, la religion est appréhendée dans sa mobilité : circulation des rituels, des pratiquants, des chefs religieux, des compétences et des savoirs, des objets de cultes, des saints, des esprits ou des breuvages sacrés. Mais derrière ces modalités de circulation aujourd'hui extrêmement répandues, se cachent de véritables constructions religieuses transnationales, souvent liées aux configurations migratoires, ainsi que des parcours et des processus fortement ancrés dans une longue histoire sociale, économique et politique.

En effet, le langage de la mobilité ne semble pas se restreindre aux seules pratiques religieuses contemporaines [Hervieu-Léger, 1999]. Le culte de Kunde est, en ce sens, un excellent exemple de l'enracinement historique de la « mobilité religieuse » puisque, comme le montre Laurent Manière dans ce numéro, la capacité d'emprunter et d'innover en matière religieuse n'est absolument pas un 
phénomène nouveau, en Afrique comme ailleurs. Si la caractéristique centrale de cette mobilité est bien "l'itinérance pluridirectionnelle des acteurs » (Losonczy et Mesturini), cela n'est pas sans évoquer des processus plus anciens, comme les trajectoires historiques et spatiales du Kunde ou du gorovodu dans le contexte colonial, de la Gold Coast jusqu'au Dahomey. De même, comme le souligne Sylvie Pedrón Colombani dans ce numéro, on retrouve dans les traits de Maximón, devenu au Guatemala le protecteur de ceux qui voyagent pour subvenir aux besoins de leurs familles, les traces d'Ek Chuah, le dieu noir du commerce des Mayas à qui les commerçants demandaient protection afin de rentrer sains et saufs de leurs voyages. Et les efforts déployés à présent par les Cook Islanders, dont nous parle Gwendoline Malogne-Fer, afin de maintenir des liens avec les personnes restées dans les îles de Polynésie, grâce aux échanges monétaires, de biens et d'informations (téléphone, internet, cassettes), ne sont pas sans évoquer d'autres dispositifs rituels, comme le kula, sorte de phénomène de globalisation ante litteram dans cette région du monde, qui montre comment le concept d'autosubsistance, appliqué aux sociétés primitives, peut être trompeur.

Si le mouvement est aujourd'hui au cœur de la «modernité religieuse », cette mobilité n'est en aucun cas une nouvelle donne, mais revisite des processus qui sont constitutifs de toute construction culturelle. Ainsi, comme le rappelle Stefania Capone dans son article, le «transnationalisme » n'est pas un nouveau phénomène, mais une «nouvelle perspective » qui permet d'appréhender des phénomènes qui, dans le passé, n'étaient pas complètement intelligibles et qui, à présent, sont amplifiés par les nouvelles technologies : facilité des déplacements, nouveaux moyens de communication, etc. Les processus de transnationalisation religieuse deviennent ainsi une clef d'analyse indispensable des réseaux migratoires, puisque l'impact des références et des espaces religieux dans la constitution ou la réorientation des trajectoires migratoires s'avère aujourd'hui particulièrement important.

Dans ce numéro, nous avons voulu réunir différentes contributions, à partir d'aires culturelles et mouvements religieux distincts. Notre principal souhait était de sortir de nos disciplines respectives pour croiser les regards et les approches disciplinaires sur des phénomènes qui interrogent à la fois les parcours migratoires et les pratiques religieuses. Les articles composant ce numéro proposent ainsi des analyses qui croisent dispositifs et pratiques religieuses dans l'étude des processus migratoires.

\section{Paysages religieux et imaginaires transnationaux}

La plupart des approches théoriques du «transnationalisme», pour reprendre le terme qui est le plus souvent utilisé dans les ouvrages anglo-saxons, n'accorde qu'un intérêt relatif aux reconfigurations, au sein du champ transnational, des paysages physiques et aux recompositions des imaginaires transnationaux. Les études sur la transnationalisation religieuse - en mettant l'accent sur les processus 
plutôt que sur les propriétés de ce phénomène - peuvent combler ce manque, en complétant les analyses classiques sur les migrations. En effet, la transnationalisation entraîne le re-façonnement des paysages religieux, puisqu'elle pose ce que Karen McCarthy Brown [1991] a appelé le « problème cosmologistique », à savoir, comment pratiquer une religion qui est liée à un ou à des lieux, lorsqu'on est ailleurs. Et lorsque les paysages physiques sont altérés, les imaginaires, eux aussi, se modifient. Si les migrants utilisent souvent la religion pour affirmer leur appartenance à plusieurs communautés, ils l'utilisent aussi pour créer des « géographies religieuses » alternatives, qui se superposent ou dépassent les frontières politiques nationales. Ces paysages religieux ne sont pas nécessairement des créations exnihilo, mais souvent des adaptations au nouveau contexte qui permettent aux migrants de reproduire des pratiques issues de leur contexte d'origine.

Cela remet en cause les analyses qui voudraient que la globalisation soit intimement liée à la perte de tout ancrage territorial, entraînée par le déplacement et le déracinement dans les parcours migratoires. Or, les recherches les plus récentes sur la transnationalisation religieuse ont montré que les processus de déterritorialisation se font rarement sans une successive reterritorialisation, grâce aussi à la production parallèle de discours sur les origines qui permettent de « réancrer » ce qui a été « déterritorialisé » dans de nouveaux espaces, réels ou symboliques $[c f$. Capone, 2004]. L'article de Mathieu Claveyrolas, ainsi que celui de Pierre-Joseph Laurent et Charlotte Plaideau, en sont des exemples particulièrement éclairants. Dans le premier article, les descendants des «engagés » hindous à l'île Maurice pallient la perte originelle du territoire indien, perçu comme paysage religieux exclusif, par un refaçonnement du paysage local qui permet d'inscrire l'île Maurice dans un «continuum hindou »(Claveyrolas). Si l'efficacité de l'hindouisme repose avant tout sur la bonne réalisation des rites, il faut alors les recréer en modifiant en profondeur les paysages religieux : l'eau du lac du Grand Bassin, rebaptisé Ganga Talao ou « lac du Gange », est ainsi censée relier les sources souterraines du fleuve sacré indien et les cendres des morts hindous-mauriciens pourront y être versées, afin qu'elles rejoignent le territoire de leurs ancêtres. Dans le deuxième article, les « esprits apatrides » du rationalisme chrétien au Cap-Vert, malgré leur position liminale, permettent de ré-ancrer l'expérience de la migration dans ce que Laurent et Plaideau appellent « une société à distance », qui relie symboliquement, par un «lien sacré », les habitants des îles avec les migrants, ainsi que les migrants entre eux.

Cependant, les pratiques transnationales se déroulent rarement dans un espace imaginaire, situé dans un « entre-deux ». Le transnational est, au contraire, fortement ancré dans le local, bien qu'il porte en soi les marques du global. De même, au lieu d'affaiblir les liens avec les origines, les mouvements transnationaux renforcent et revitalisent parfois les discours sur les origines en redéfinissant les territoires religieux. Ces territoires ne sont pas liés exclusivement à des lieux de cultes établis le long des routes de la migration (Odgers, Bava et Picard), mais aussi à des objets de culte qui circulent, des saints-voyageurs (Pedrón Colombani), des images pieuses et autres objets que l'on amène avec soi. Tout un dispositif 
religieux est mis en place par la circulation d'objets, d'hommes ou d'esprits (Laurent et Plaideau), c'est-à-dire des éléments qui sont constitutifs de l'identité du migrant et qui recréent une continuité territoriale entre les différents lieux de la migration. Certains réseaux religieux sont même à l'origine des mobilités et souvent même des migrations, comme les réseaux d'études islamiques entre Afrique noire et monde arabe [Bava et Pliez, 2009] ou les réseaux catholiques qui aident l'accueil et le placement de certains migrants dans des niches d'emploi particulières, telles que le travail domestique.

La question du territoire religieux, qui s'étend à travers la circulation des migrants et qui permet parfois de faire émerger une communauté transnationale, est très présente dans ce numéro et est étroitement liée à la persistance d'imaginaires nationaux au sein des constructions religieuses transnationales. Le cas des colons-migrants de la communauté hindoue de l'île Maurice est en ce sens exemplaire, puisqu'ils ont su ancrer leurs pratiques religieuses et développer un territoire religieux qu'ils inscrivent dans une continuité territoriale avec des lieux sacrés en Inde, légitimant ainsi leur identité hindoue-mauricienne. De même, l'article de Fortunato Mallimaci et Veronica Giménez Béliveau met en lumière la redéfinition des imaginaires locaux et transnationaux face aux migrations dans la région de la «Triple Frontière », entre l'Argentine, le Paraguay et le Brésil, en faisant de l'Autre migrant un « Autre religieux ». La «Triple Frontière » est alors construite comme un «territoire transnational », au sein duquel est activé un imaginaire fortement marqué par des idées nationalistes et qui renvoie à des projets politiques régionaux. Ceci nous permet de penser la « logique de distinction inclusive » - soulignée par Ulrich Beck [2006, p. 132] dans son analyse de la «transnationalité » des Hmong «américains »- de ce que nous pouvons appeler un «nationalisme transnational». Comme le rappellent Stefania Capone et André Mary [2011], ce concept, qui n'est pas sans évoquer les « transnations indigènes » d'Appadurai [2005], permet aux acteurs de refuser de s'enfermer dans les frontières de l'État-nation sans pour autant s'en exclure complètement. Les imaginaires transnationaux réinventent ainsi des « Nations premières » qui ont une vocation paradoxale à fournir, par le biais de leur particularisme, des vecteurs d'universalité [Capone et Mary, 2011].

Cela remet en cause l'idée que la globalisation et la transnationalisation affaiblissent automatiquement le rôle de l'État-nation. Plusieurs cas évoqués dans les articles réunis dans ce numéro montrent comment le rôle joué par les appartenances nationales s'avère, au contraire, très important dans la transnationalisation des pratiques religieuses. Ainsi la Vierge d'Urkupiña, symbolisant « l'intégration nationale » dans une Bolivie en proie à de fortes tensions régionales, devient le symbole d'une «bolivianité diasporique » dans les territoires de la migration bolivienne (Baby-Collin et Sassone), tout comme la divinité guatémaltèque Maximón, devenue San Simón à Los Angeles, se métamorphose, lors de sa re-localisation, en l'incarnation d'une «dimension indienne générique » (Pedrón Colombani). Ses célébrations en Californie rassemblent un grand nombre de Centre-Américains "se déguisant en Guatémaltèques", ce qui semble révéler une sorte de 
«pan-latinisation » et la création de «publics transnationaux » dans des pratiques religieuses qui s'adressent à des communautés latinas «transnationales » [Vasquez, 1999].

Mais la migration renforce également les religions d'origine et les territoires religieux des pays de départ, comme nous pouvons le voir dans certains articles (Laurent et Plaideau, Mesturini et Losonczy, Pédron Colombani, Odgers), ou encore les reconfigure lorsque les migrants, en rentrant dans leur pays d'origine, rapportent avec eux une attitude de « tolérance » vis-à-vis de la diversité religieuse rencontrée dans les pays d'accueil. Les articles de Maïa Guillot sur la transnationalisation des religions afro-brésiliennes au Portugal et d'Olga Odgers sur le paysage religieux mexicain montrent comment les processus de transnationalisation se mettent en place dans les deux directions : s'ils ont un effet certain sur les sociétés d'accueil, ils laissent aussi leur empreinte sur les sociétés d'origine, puisque les migrants rapportent avec eux des nouvelles façons de pratiquer leur religion, qui modifient la pratique traditionnelle dans leurs communautés de départ.

\section{Des acteurs, clefs de voûte des dispositifs religieux transnationaux}

Les statuts sociaux des différents «migrants », abordés dans ce numéro thématique, sont, pour le moins, différents : migrants économiques, réfugiés, étudiants, « engagés », déplacés, missionnaires, colons, migrants-colons, migrants de retour, toute une gamme de statuts qui auront, comme nous allons le voir, une influence certaine dans le rapport que ces hommes et femmes entretiennent avec le religieux. En témoigne l'ascension sociale rapide des descendants des « engagés » hindous à l'île Maurice qui, selon Claveyrolas, leur aurait permis de maintenir vivace la mémoire de leur culture religieuse et de la reterritorialiser. D'autres migrants, comme les Cooks-Islanders en Nouvelle Zélande (MalogneFer), les Africains au Caire (Bava et Picard) ou les Portugais rentrés au pays après avoir émigré au Brésil (Guillot), découvrent une véritable vocation religieuse en reinterprétant leurs expériences migratoires. La dimension «missionnaire » est alors souvent évoquée pour justifier les parcours migratoires. Tous ces acteurs évoluent dans un contexte transnational. Ils se pensent et vivent quotidiennement en tant que membres d'un groupe qui se constitue dans un espace interstitiel ou transversal [Levitt, 2004], en développant une conscience d'appartenir à deux mondes à la fois, à leur terre d'origine ainsi qu'à leur terre d'accueil.

Figures insolites de la migration, les acteurs religieux n'occupent pas moins une place essentielle dans l'histoire des migrations. Dans les parcours migratoires, l'identité religieuse peut apparaître comme une valeur refuge aux contours fragiles. Marqués par ces identités fragmentées, de nombreuses figures de migrants émergent: les pèlerins, les guérisseurs, les cheikh-s itinérants à la rencontre des migrants, les pasteurs missionnaires et les migrants devenus pasteurs, les commerçants, les étudiants religieux, les initiés entre plusieurs territoires ou les saints que l'on vient consulter avant d'émigrer afin d'obtenir leur bénédiction et leur 
protection. Toute une gamme d'acteurs religieux est abordée dans ces articles, éléments primordiaux dans la compréhension de ces processus.

Toutes ces figures d'acteurs sont aussi des « acteurs-passerelle », qui permettent de mettre en contact des cultures et des pratiques religieuses distinctes. Cette possibilité de traduire des localités diverses - ou de « glocaliser » la pratique est indispensable pour pouvoir penser les modalités d'adaptation et résoudre les possibles conflits avec les traditions locales. Mais suivre les acteurs, comme le rappelle Stefania Capone dans ce numéro, signifie aussi modifier en profondeur son approche du terrain, qui requiert un engagement à long terme. Si l'essence de la recherche sur un terrain transnational est de «savoir circuler » [Tarrius, 2000], en suivant les parcours des acteurs, il est aussi indispensable de savoir manier l'ensemble des références, des savoirs, des pratiques et des langages qui fondent ces espaces sociaux transnationaux, en développant une véritable « ethnographie multilangages ou multicodes ». Privilégier les acteurs signifie aussi adopter une approche en termes de « champ social transnational », qui est constitué par les multiples réseaux de relations sociales, à travers lesquels des pratiques, des idées, des ressources sont réorganisées, transformées et échangées de façon inégale. Ce paradigme permet ainsi d'appréhender les enjeux politiques et les rapports de pouvoir au sein des processus transnationaux.

\section{Logiques duales et dynamiques religieuses}

La migration entraîne également la réactivation et la revitalisation de lieux de cultes, ainsi que de nouvelles vocations religieuses et conversions. Il ne s'agit pas seulement de cultes transplantés par les migrants, mais aussi de mouvements religieux nouvellement créés par eux. Les lieux qui les accueillent deviennent alors des lieux de concurrence dans l'offre religieuse : que ce soit au Caire ou au Portugal, l'installation des migrants produit une offre religieuse qui est souvent en concurrence avec celle déjà existante. Les migrations créent ainsi les conditions d'émergence d'un marché religieux renouvelé dans lequel plusieurs mouvances se confrontent, en combinant prétentions à l'« authenticité » et ambiguïté.

En effet, l'un des ressorts principaux des entreprises transnationales est la dualité de l'expérience religieuse, qui reprend les «dual lives», les « vies doubles », vécues par les immigrés entre leur terre d'origine et leur pays d'accueil. Stefania Capone et André Mary [2011] ont souligné les logiques duales et paradoxales qui fondent le fonctionnement de la transnationalisation religieuse, en parlant de «trans-logiques» pour penser les logiques de déplacement et de conservation d'une identité dédoublée, ainsi que la tension et la cohabitation entre des tendances antagonistes ou contradictoires : homogénisation vs fragmentation ; universalisation vs particularisme local ; syncrétisme vs anti-syncrétisme, etc. Or, comme il a été souligné par ces auteurs [ibid.], l'hybridité pourrait être la clef de la transportabilité pratique [cf. Csordas, 2009] de certaines religions transnationales. 
Les articles réunis dans ce numéro montrent bien comment, à l'origine d'une relocalisation, on trouve souvant des figures duales, médiatrices et ambiguës. C'est le cas, par exemple, de la «Vierge prêteuse» (Virgen prestamista) dont nous parlent Virginie Baby-Collin et Susana Sassone, à laquelle les pèlerins « empruntent » des pierres taillées dans la montagne, qu'ils lui restitueront lorsque le vœu de leur réussite économique sera exaucé. Ce n'est pas un hasard si le culte à cette vierge est installé dans une région marquée par une tradition de mobilité ancienne, qui en fait depuis longtemps une figure médiatrice dans les parcours migratoires des habitants de Cochabamba et du Valle Alto. Ou encore le cas de Mam/ Maximón/San Simón, multiples visages d'une même divinité qui se transforme en des figures différentes selon les échelles et les étapes des migrations guatémaltèques. Si le dieu maya Mam, premier avatar de Maximón, était déjà intimement lié au renouvellement du cycle vital, Maximón est, quant à lui, lié à la nouvelle vie dans un nouveau pays. Sylvie Pedrón Colombani souligne l'ambiguité de ce personnage, sa dualité, qui lui permet d'incarner, à San Andrés Itzapa, une identité «maya » sans frontières, tout en se déguisant en personnage occidental (peau blanche, costume occidental...), alors que, à Los Angeles, il deviendra un Italien, en épousant la réalité et les préoccupations des migrants dans une nouvelle société. Il en est de même pour la position ambiguë occupée par le gorovodu face au christianisme et sa situation interstitielle dans l'espace religieux (Manière), pour la logique hybride des « esprits apatrides » du Cap-Vert (Laurent et Plaideau) ou encore pour celle des esprits brésiliens, mais aussi depuis quelque temps portugais, dans les maisons de culte d'umbanda et de candomblé au Portugal (Guillot). D'autres auteurs soulignent le rôle de médiation d'objets ou de substances, comme l'ayahuasca qui est définie comme une «substance médiatrice », « métonymie mobile de la forêt amazonienne et du monde indigène », une substance qui était déjà ancrée dans une tradition d'origine précoloniale de circulation interethnique d'objets, de savoirs et d'apprentis chamanes (Losonczy et Mesturini).

Ainsi, ce qui se transnationalise est, souvent, déjà un élément «médiateur » dans son contexte d'origine. C'est autour de ces éléments médiateurs que se construisent des nouvelles cartographies religieuses, dans lesquelles les « acteurspasserelle » - les passeurs entre une société et l'autre - jouent, nous l'avons vu, un rôle fondamental. Mais, si l'ambigüité est au cœur de ces processus, d'autres processus semblent aussi centraux. Anne-Marie Losonczy et Silvia Mesturini parlent du «malentendu » en tant qu'élément constitutif du champ relationnel transnational. Ainsi, si le chamane local, Don Bartolomeo, se définit lui-même comme un métis, il incarne aux yeux des touristes l'image même de l'altérité indienne exotique. Son mutisme face à des Occidentaux avides d'expériences mystiques exotiques sera pallié par le Bachelor en anthropologie, Wilson, qui fonctionne comme «personnage-passerelle », un médiateur qui rend possible par sa présence la rencontre de deux perspectives souvent incompatibles.

En traitant des confrontations, des adaptations, des négociations mais aussi des malentendus qui se produisent pendant les rituels transnationalisés, Kali Argyriadis et Stefania Capone [2009] ont également montré comment le 
«malentendu » constitue l'une des voies possibles pour le «dialogue » entre modalités de culte, dont les univers de référence ne sont pas nécessairement « traduisibles » les uns dans les autres. Dans ce cas, il s'agit de «malentendus productifs » ou «working misunderstandings » [Sahlins, 1985], puisqu'ils rendent possible la gestion de la contiguïté, qui peut alors utiliser le malentendu comme ressource. Cependant, les résultats différeront selon l'habilité des acteurs en présence à mener cette négociation entre matrices de sens. Les « acteurs-passerelle » sont, en ce sens, indispensables à la gestion des malentendus et de la rencontre culturelle.

\section{Conclusion}

Ce numéro a pour ambition de sortir de l'opposition, prévalente en France, entre spécialistes des religions et spécialistes des migrations, en ouvrant de nouvelles pistes de réflexion dans l'analyse des croisements entre religion et migration. La variété des articles que nous avons reçus montre bien que ce thème de recherche traverse les frontières de nos disciplines et de nos spécialités. Si la spécialisation qu'atteignent les anthropologues des religions en suivant certains cultes est primordiale, elle doit aussi être combinée à la compréhension des processus migratoires et de leurs acteurs. C'est le cas dans les études sur la transnationalisation des religions portées par des migrants, bien que ces études ne se limitent pas, rappelons-le, aux seuls processus migratoires.

Quels que soient les articles et l'entrée choisis, les auteurs ont tous pris le parti d'étudier les pratiques religieuses en migration comme des expressions de processus religieux globaux et non comme des actes isolés dans le temps et l'espace de la migration. L'intensification des migrations n'engendre donc pas une religion à la carte déterritorialisée. Au contraire, elle est créatrice de continuités entre plusieurs mondes et de mouvements religieux qui empruntent à la fois à l'histoire, à la mémoire et aux rencontres nées de la mobilité.

\section{Bibliographie}

Appadurai A. [2005], Après le colonialisme. Les conséquences culturelles de la globalisation, Paris, Petite Bibliothèque Payot (Édition originale, 1996, Modernity at large : Cultural Dynamics of Globalization, Minneapolis, University of Minnesota Press).

ANDEZIAN S. [1983], « Pratiques féminines de l'islam en France », Archives de Sciences Sociales de Religions, vol. 55, $\mathrm{n}^{\mathrm{o}}$ 1, janvier-mars, p. 53-66.

ANDEZIAn S. [2001], Expériences du divin dans l'Algérie contemporaine. Adeptes des saints dans la région de Tlemcen, Paris, CNRS Éditions.

ARgYriAdis K., CAPONE S. [2009], «Adaptations rituelles et malentendus productifs : la transnationalisation des religions afro-cubaines (Veracruz, Rio de Janeiro) », Communication présentée au Colloque international «Transnationalisation religieuse des Suds : entre ethnicisation et universalisation », Programme ANR-RELITRANS, Bondy (France). 
BAROU J. [1978], Travailleurs africains en France, Grenoble, Presses universitaires de Grenoble.

Basch L., Glick-Schiller N., Szanton Blanc C. [1994], Nations Unbound. Transnational Projects, Post-colonial Predicaments, and De-territorialized Nation-States, Langhorne, PA, Gordon and Breach.

Bauberot J. (dir.) [1994], Religion et laïcité dans l'Europe des douze, Paris, Syros.

BAVA S. [2002], « Itinéraires religieux et routes migratoires. Des pratiques religieuses des Sénégalais mourides entre Marseille et Touba », Thèse de doctorat, EHESS.

BAVA S. [2003], «De la baraka aux affaires : ethos économico-religieux et transnationalité chez les migrants sénégalais mourides », Revue Européenne des Migrations Internationales, vol. $19, \mathrm{n}^{\circ} 2$, p. 69-84.

Bava S. [2011], «Research on the Connection between Migration and Religion in France : Evolving Toward a Religious Anthropology of Movement », Annual Review of Anthropology, vol. 40 (à paraître).

BAVA S., Pliez O. [2009], « D 'Al Azhar à l'économie de bazar. Itinéraires socio-économiques des "élites musulmanes" africaines au Caire», Afrique Contemporaine, vol. 3, n 231, p. 187-207.

BECK U. [2006], Qu'est-ce que le cosmopolitisme?, Paris, Aubier Alto.

Bonte P., Brisebarre A.-M., Gokalp A. [1999], Sacrifice en islam. Espace et temps d'un rituel, Paris, CNRS Editions.

CAPONE S. [2004], «A propos des notions de globalisation et transnationalisation », numéro spécial « Religions transnationales », Civilisations, Bruxelles, vol. LI, nº 1-2, p. 9-22.

CAPONE S., MARY A. [2011], «Les translogiques d'une globalisation religieuse à l'envers », in Argyriadis K., Capone S., de la Torre R., Gutierrez C., Mary A. (dir.), Religions transnationales : entre ethnicisation et universalisation, Paris (à paraître).

CESARI J. [1994], Être musulman en France. Associations, militants et mosquées, Paris, Editions Karthala et Iremam.

CsoRdas T. (dir.), Transnational Transcendence. Essays on Religion and Globalization, Berkeley, University of California Press.

DAsSETto F., BASTENIER A. (dir.) [1991], Immigrations et nouveaux pluralismes, Bruxelles, Ed. De Boeck Wesmael.

Diop A. M. [1989], «Immigration et religion : les musulmans négro-africains en France », Migration-sociétés, numéro 5-6, p. 45-57.

Diop A. M., Kastoryano R. [1991], «Le mouvement associatif islamique en Ile de France », Revue Européenne des Migrations Internationales, vol. 7, nº 3, p. 91-117.

FANCELlo S. [2005] «Réseaux migratoires et structures de pouvoir : la Church of Pentecost face aux communautés francophones », in Fourchard L., Mary A., Otayek R. (dir.), Entreprises religieuses transnationales en Afrique de l'Ouest, Paris, IFRA-Karthala, p. 113-136.

FANCELLO S. [2006], Les aventuriers du pentecôtisme ghanéen. Nation, conversion et délivrance en Afrique de l'Ouest, Paris, IRD-Karthala.

GeISSER V. [2001], « Marseille, capitale de l'islam méridional », La Medina, nº 7, p. 24-32.

Glick-Schiller N., Basch L., Szanton BlanC C. [1995], « From Immigrant to Transmigrant : Theorizing Transnational Migration », Anthropological Quarterly, vol. 68, n 1, p. 48-63.

Guarnizo L. E. [1997], « The Emergence of a Transnational Social Formation and the Mirage of Return among Dominican Transmigrants », Identities, nº 4, p. 281-322. 
Hervieu-Leger D. [1999], La religion en mouvement: le pèlerin et le converti, Paris, Flammarion.

KUCZYNSKi L. [2002], Les marabouts africains à Paris, Paris, CNRS Editions.

KUCZYNSKi L., RAZY E. [2009], « Anthropologie et migrations africaines en France. Une généalogie des recherches », Revue Européenne des Migrations Internationales, vol. 25, $\mathrm{n}^{\circ} 3$, p. $79-100$.

LevitT P. [2004], «Redefining the Boundaries of Belonging: The Institutional Character of Transnational Religious Life », Sociology of Religion, vol. 65, nº 1, p. 1-18.

MARY A. (dir.) [2008], «Christianisme du Sud à l'épreuve de l'Europe », Archives de sciences sociales des religions, $\mathrm{n}^{\circ} 143$.

McCarthy Brown K. [1991], Mama Lola : A Vodou Priestess in Brooklyn, Berkley, University of California Press.

Quiminal C. [1991], Gens d'ici, gens d'ailleurs. Migrations soninké et transformations villageoises, Paris, Bourgeois.

SAHLIns M. [1985], Islands of History, Chicago, University of Chicago Press (Trad. fr. Des îles dans l'histoire, Paris, EHESS/Gallimard/Seuil, 1989).

Saint-Blancat C. [1995], "Une diaspora musulmane en Europe », Archives de Sciences Sociales des Religions, ${ }^{\circ} 92$ (octobre-décembre), p. 9-24.

Saint-Blancat C. [1997], L'islam de la diaspora, Paris, Bayard Editions.

TARrius A. [1989], Anthropologie du mouvement, Caen, Paradigme.

TARRIUS A. [1996], «Territoires circulatoires et espaces urbains », Annales de la Recherche Urbaine, $\mathrm{n}^{\circ}$ 59-60, p. 50-59.

TARrius A. [2000], Les nouveaux cosmopolitismes. Mobilités, identités, territoires, La Tour d'Aigue, Editions de l'Aube.

VASQUeZ M. [1999], « Pentecostalism, Collective Identity, and Transnationalism among Salvadorans and Peruvians in the U.S. », Journal of the American Academy of Religion, vol. 67, $\mathrm{n}^{\mathrm{o}} 3$, p. 617-636. 